\title{
Estimativa de Projetos de Aplicativos Móveis: Um Mapeamento Sistemático da Literatura
}

\author{
Ervili T. B. de Souza ${ }^{1}$, Tayana Conte ${ }^{1}$ \\ ${ }^{1}$ Grupo de Pesquisa em Usabilidade e Engenharia de Software (USES) - Instituto de \\ Computação - Universidade Federal do Amazonas (UFAM) \\ Av. Rodrigo Otávio, nº 6.200. CEP: 69077-000, Manaus - AM - Brasil \\ \{etbs, tayana\} @icomp.ufam.edu.br
}

\begin{abstract}
Mobile applications have needs and characteristics different from other information systems. These characteristics are factors that influence the effort estimation. In this context, this paper presents a systematic literature mapping about characteristics of effort predictor factors and estimation models for mobile application projects. As a result, we identified five models for size estimation, three models for effort estimation, three models for estimating size and effort together and ninety-three predictor factors. From the results, we conclude that there is no standardization of effort predictor factors that support professionals to estimate.
\end{abstract}

Resumo. As aplicações móveis têm necessidades e características diferentes em relação aos demais sistemas de informação. Essas características são fatores que influenciam a estimativa de esforço. Neste contexto, este artigo apresenta um mapeamento sistemático da literatura que caracteriza fatores preditores de esforço e modelos de estimativa voltados para projetos de aplicativos móveis. Como resultado, foram identificados cinco modelos para estimar tamanho, três modelos de esforço, três modelos para estimar tamanho e esforço em conjunto e noventa e três fatores preditores. A partir dos resultados, conclui-se que não há uma padronização dos fatores preditores de esforço que apoie os profissionais a estimar aplicativos móveis.

\section{Introdução}

A eMarketer (2016) estima que existam mais de 2,16 bilhões de clientes móveis em todo o mundo, ou seja, mais de um quarto da população mundial utiliza smartphones, prevendo um maior crescimento no uso desta tecnologia nos próximos anos. Com a evolução dos dispositivos móveis, os usuários foram motivados a incorporar os aplicativos aos seus dispositivos para ajudá-los a realizar suas atividades diárias, como comunicação, negócios, esportes, educação e notícias.

Um aplicativo móvel é definido como o aplicativo desenvolvido para a geração atual de dispositivos móveis, popularmente conhecidos como smartphones [NAGAPPAN 2016]. De acordo com Dombroviak e Ramnath (2007), as aplicações móveis têm necessidades e características diferentes que os sistemas de informação tradicionais, tais como: centricidade (coleta de dados ou execução de ações em resposta 
ao ambiente), consciência de localização absoluta (usam tipicamente métodos como GPS ou triangulação de sinal), consciência de aproximação (sensibilidade de aproximação de determinado objeto e espaço), consciência de espaço (o dispositivo está dentro da área de limite definido de lugares, exemplos: edifícios, prédios e etc.) e consciência de transição (o grau em que o comportamento de uma aplicação depende do conhecimento de transições entre espaços). Além dessas, De Souza e Aquino (2014) complementam com as seguintes características: consumo de bateria, tipos de conectividade, fator de desempenho e outros. Segundo Nitze et al. (2014), essas características são fatores influentes (ou restrições) que modificam a estimativa de esforço, devido a isto, determinados processos como a estimativa de esforço devem ser adaptados para este novo contexto. Os processos de estimativa são baseados em características de sistemas que quantificam a complexidade de implementá-los [DE SOUZA e AQUINO 2014].

Considerando a importância que o desenvolvimento de aplicativos móveis desempenha na indústria de hoje e suas diferentes características comparada a sistemas de software em geral, um mapeamento sistemático da literatura é importante para estabelecer o estado atual de como é realizada a estimativa de esforço de aplicativos móveis. Este mapeamento sistemático foi orientado a identificar, avaliar e interpretar todas as pesquisas disponíveis relevantes [KITCHENHAM e CHARTES 2007].

Além desta seção introdutória, as seções seguintes deste artigo estão organizadas da seguinte forma: A Seção 2 apresenta o referencial teórico sobre conceitos de estimativa de projetos. A Seção 3 detalha o protocolo do mapeamento sistemático e o método de pesquisa utilizado. A Seção 4 apresenta os resultados do mapeamento para cada questão de pesquisa. A Seção 5 discute os resultados encontrados sobre estimativa de tamanho, esforço e fatores preditores de esforço de aplicativos móveis. Por fím, a Seção 6 apresenta conclusões e trabalhos futuros.

\section{Referencial Teórico}

Segundo Mendes (2014), estimar em Engenharia de Software consiste em prever prazo, recurso e esforço necessários para desenvolver um conjunto de tarefas do ciclo de vida de um projeto de software. Abreu (2011) divide a estimativa de projetos de software em três tipos:

- Estimativa de Tamanho - Grandeza física medida através dos requisitos, análise e projeto ou código do software com base nas suas funções e complexidade do problema.

- Estimativa de Esforço - Trabalho necessário para o desenvolvimento do projeto obtido a partir da estimativa de tamanho.

- Estimativa de Prazo - Tempo necessário para o desenvolvimento do projeto obtido a partir da estimativa de esforço e quantidade de recursos envolvidos no projeto

Mendes (2014) divide as categorias de estimativa em três tipos: estimativa baseada por especialistas que é o processo de estimativa fundamentada na experiência no desenvolvimento ou gestão de projetos anteriores, técnicas algorítmicas que 
constroem modelos que representam precisamente a relação entre esforço e características do projeto através do uso de modelos algorítmicos (um exemplo é o COCOMO [BOEHM 1981]) e técnicas de inteligência artificial que são usadas como um complemento para as duas categorias anteriores. Além disso, Mendes (2007) afirma que fatores preditores de esforço são métricas de tamanho e fatores de custo.

Em relação a estimativa de aplicativos móveis, De Souza e Aquino (2014) realizaram uma revisão sistemática da literatura sobre as características das aplicações móveis. Do conjunto de 234 artigos identificados, 40 foram aceitos por meio dos critérios definidos por eles e 29 características foram extraídas. Depois, os autores realizaram um refinamento dessas características por meio de um survey nas universidades e empresas que desenvolvem aplicativos móveis com a finalidade de ratificar as características previamente levantadas e para provar sua respectiva influência sobre o desenvolvimento móvel, resultando em 13 características. Porém, o foco deste mapeamento sistemático é investigar como é realizada a estimativa de aplicativos móveis e não em características especificas de aplicativos móveis. A seção a seguir apresenta o protocolo do mapeamento sistemático da literatura realizado pelas autoras deste artigo.

\section{Mapeamento Sistemático da Literatura}

\subsection{Questões de Pesquisa}

Uma abordagem para a formulação das questões de pesquisa é utilizar os critérios especificados pelo PICOC [KITCHENHAM E CHARTERS 2007] que investiga as estruturas das perguntas de acordo com cinco atributos: população, intervenção, comparação, resultados e contexto. No entanto, o foco deste mapeamento da literatura é caracterizar intervenções, logo os atributos de comparação não serão utilizados e, portanto, apenas atributos como a população, intervenção e resultado (PIO) serão considerados. A Tabela 1 mostra os detalhes.

Tabela 1. Questões de investigação estruturadas pelos critérios do PIO

\begin{tabular}{|l|l|}
\hline População & Projetos de Aplicativos Móveis \\
\hline Intervenção & $\begin{array}{l}\text { Métodos / técnicas/ modelos utilizados para a estimativa de tamanho e/ou } \\
\text { esforço para aplicativos móveis, fatores preditores considerados. }\end{array}$ \\
\hline Resultado & $\begin{array}{l}\text { Métodos / técnicas/ modelos utilizados para aplicativos móveis e fatores } \\
\text { preditores. }\end{array}$ \\
\hline
\end{tabular}

A questão de pesquisa foi formulada para que pudesse abranger todo o escopo de estimativa de projetos de aplicativos móveis em Engenharia de Software. Neste contexto, a questão de pesquisa deste mapeamento sistemático é a seguinte: "Como é realizada a estimativa de projetos de aplicativos móveis?". A partir dessa questão de pesquisa foram definidas três subquestões:

- Que modelos e métodos têm sido utilizados para a estimativa de tamanho e esforço de aplicativos móveis?

- Como foram avaliados os modelos e métodos de estimativa? 
- Que fatores têm sido investigados como preditores de esforço para Aplicativos Móveis?

\subsection{Termos de Pesquisa}

Os termos de pesquisa foram reunidos em uma string de busca que foi utilizada no processo de pesquisa. Os termos estão escritos em inglês por ser adotado pela grande maioria das conferências e periódicos internacionais relacionados ao tema de pesquisa. A seguir está a string de busca, sendo que foi utilizado um recurso oferecido pelas fontes de busca que delimita a área de pesquisa Ciência da Computação.

\section{("Mobile apps" OR "Mobile Applications" OR "Mobile Computing" OR "Mobile System" OR "Mobile Software") AND ("Process" OR "Technique" OR "Model" OR "Method" OR \\ "Approach" OR "Features" OR "Characteristics" OR "Factors") AND ("Effort Estimat*" OR "Effort Prediction" OR "Effort Measurement" OR "Size Estimat*” OR "Functional Size Measurement")}

Para avaliar a qualidade e abrangência da string de busca, foi realizada uma pesquisa exploratória na qual foram definidos 4 artigos de controle, que são Nitze et al. (2014), De Souza e Aquino (2014), Heeringen e Gorb (2014) e Ferrucci et al. (2015b). Após a execução da string de busca nas bibliotecas digitais, verificou-se que os artigos de controle estavam entre as publicações retornadas.

\subsection{Fontes de Busca}

A busca foi realizada nas bibliotecas digitais Scopus, Engineering Village e ACM, pois as três bibliotecas digitais possuem um bom funcionamento e abrangência de suas máquinas de busca, evidenciada em alguns trabalhos, como o de Nitze et al. (2014), Francese et al. (2015) e Pocatilu e Vetrici (2009). Kitchenham e Charters (2007) afirmam que a Scopus é a maior base de dados de indexação de resumos e citações. A ACM DL também indexa algumas publicações da Springer Link, Science Direct. Trindade et al. (2008) afirmam que Engineering Village agrega informações de diversos bancos de dados bibliográficos em Ciência da Computação (Compendex e Referex), abrangendo importantes periódicos e conferências da IEEE, ACM, Springer e Elsevier.

\subsection{Critérios de Seleção de Estudos}

A fim de selecionar os estudos que melhor respondem as questões de pesquisa, Kitchenham e Charters (2007) sugerem a definição de critérios de inclusão e exclusão para os estudos que são retornados pela string de busca. Para este mapeamento, foram definidos os critérios descritos na Tabela 2. Não foi adicionado critério de exclusão de artigos que não fossem relacionados a artigos primários com o objetivo de abranger estudos secundários e terciários, pois nenhuma revisão ou mapeamento da literatura específico sobre estimativa de aplicativos móveis foi retornado nas buscas.

\subsection{Processo de Seleção de Estudos}

O mapeamento sistemático foi conduzido por duas pesquisadoras e o processo de seleção de estudos foi sistematizado seguindo três etapas: (1) execução da busca; (2) primeiro filtro e (3) segundo filtro. 
Durante a execução de busca, foram realizados quatro refinamentos na string de busca para obter melhores resultados. Não houve restrição no período de tempo, porém a busca do último refinamento da string ocorreu em novembro de 2016. Utilizou-se a string de busca nas fontes selecionadas e armazenou-se o conjunto de referências recuperadas na ferramenta Start $^{1}$.

Tabela 2. Critérios de Seleção

\begin{tabular}{|c|c|c|}
\hline \# & Código & Descrição \\
\hline \multirow{2}{*}{$\begin{array}{l}\text { Critérios } \\
\text { de } \\
\text { Inclusão }\end{array}$} & CI01 & $\begin{array}{l}\text { O artigo analisa a estimativa de esforço e/ou tamanho dentro do domínio do } \\
\text { desenvolvimento de Aplicativos Móveis. }\end{array}$ \\
\hline & CI02 & $\begin{array}{l}\mathrm{O} \text { artigo caracteriza os fatores preditores de esforço no desenvolvimento de } \\
\text { Aplicativos Móveis. }\end{array}$ \\
\hline \multirow{6}{*}{$\begin{array}{l}\text { Critérios } \\
\text { de } \\
\text { Exclusão }\end{array}$} & CE01 & $\begin{array}{l}\text { O artigo não analisa a estimativa de esforço e/ou tamanho dentro do domínio } \\
\text { do desenvolvimento de Aplicativos Móveis. }\end{array}$ \\
\hline & CE02 & O artigo não realiza a estimativa nas fases iniciais do projeto. \\
\hline & CE03 & $\begin{array}{l}\text { O artigo estima o tamanho do espaço de armazenamento que o aplicativo irá } \\
\text { ocupar. }\end{array}$ \\
\hline & CE04 & Artigos duplicados. \\
\hline & CE05 & Falta de disponibilidade do artigo para download. \\
\hline & CE06 & Artigos que não estão escritos em Inglês ou em Português. \\
\hline
\end{tabular}

No primeiro filtro, foram analisados os artigos retornados na busca por meio dos critérios de inclusão e exclusão, através da leitura dos títulos e abstracts dos artigos. No segundo filtro, foi realizada a leitura completa dos artigos incluídos no primeiro filtro e analisados novamente por meio dos critérios de inclusão e exclusão. Foram excluídos os artigos cujo conteúdo não atendia aos critérios de seleção, justificando a decisão. Após a verificação dos resultados da seleção do segundo filtro, foi realizada a extração dos dados dos artigos incluídos. Os resultados quantitativos obtidos em cada etapa do procedimento de seleção dos artigos são resumidos na Figura 1.

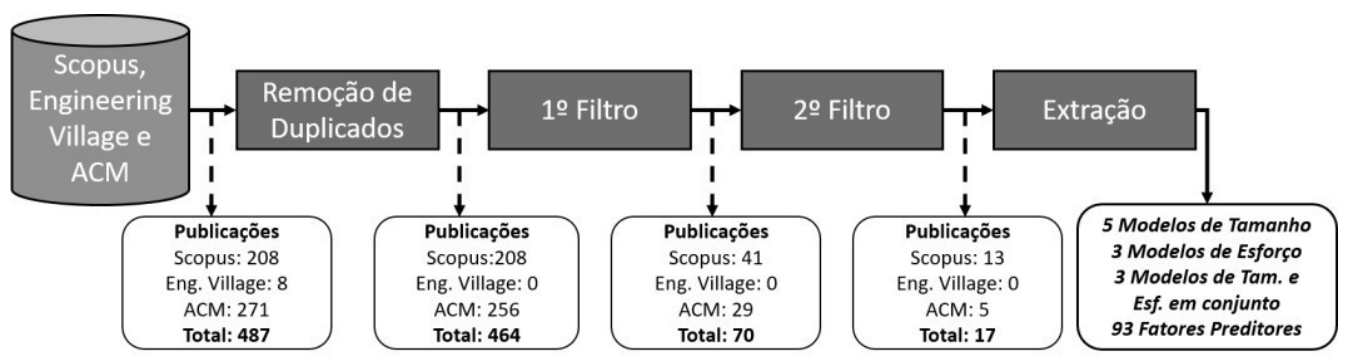

Figura 1.Processo de Seleção de Publicação

Durante a execução da busca, foram retornados 487 artigos nas fontes de busca. Em seguida, foram removidos os artigos duplicados, o primeiro filtro foi realizado por uma pesquisadora que analisou cada artigo, supervisionado pela outra pesquisadora. Foi conduzido um teste na biblioteca Engineering Village, porém 100\% das publicações foram duplicadas em relação aos resultados da Scopus. A lista de todas as publicações extraídas está disponível no Relatório Técnico [DE SOUZA e CONTE 2017].

\footnotetext{
${ }^{1} \mathrm{http}: / /$ lapes.dc.ufscar.br/tools/start_tool
} 
Com intuito de garantir a confiabilidade do processo de seleção, uma amostra de 50 artigos, selecionados aleatoriamente, foi utilizada para analisar o grau de concordância entre as duas pesquisadoras durante a seleção dos artigos. $\mathrm{O}$ acordo entre estas duas pesquisadoras foi avaliado utilizando-se o método estatístico Teste Kappa [COHEN 1960]. O resultado obtido foi um nível de concordância igual a 0,729, indicando uma concordância significativa entre as pesquisadoras [LANDIS \& KOCH 1977].

\subsection{Procedimento de Extração dos Dados dos Artigos Selecionados}

Depois do término do segundo filtro, os artigos selecionados foram submetidos ao processo de extração dos dados. A extração foi realizada de forma sistemática por meio de um formulário definido para registrar as informações necessárias relativas a cada artigo. $\mathrm{O}$ formulário de extração permite o registro de todas as informações necessárias para responder às questões de pesquisa. $\mathrm{O}$ modelo do formulário de extração encontra-se no relatório técnico [DE SOUZA e CONTE 2017].

\section{Resultados}

\subsection{Visão Geral dos Resultados}

No primeiro momento, os resultados do mapeamento sistemático foram analisados quantitativamente em relação aos veículos e ao ano de publicação. Em relação aos veículos de publicação, $11 \%$ dos artigos foram publicados em revistas ou periódicos, enquanto $89 \%$ foram publicados em conferências. No que se refere ao período de publicação, a Figura 2 mostra as publicações por ano. Desde 2005, a estimativa de projeto de aplicativos móveis vem sendo relatada nas publicações científicas. Uma possível explicação por não ter estudos em 2016 é que as publicações e trabalhos relevantes ainda não tinham sido indexados nas fontes de busca.

Das publicações analisadas para os projetos de aplicativos móveis, 28\% descrevem modelos de estimativa de tamanho, $6 \%$ abordam modelos de estimativa de esforço, $17 \%$ analisam fatores preditores de esforço, $11 \%$ abordam modelos de estimativa de tamanho e esforço no mesmo trabalho e $39 \%$ falavam de ambos (estimativa de tamanho, esforço e fatores preditores de esforço para projetos de aplicativos móveis).

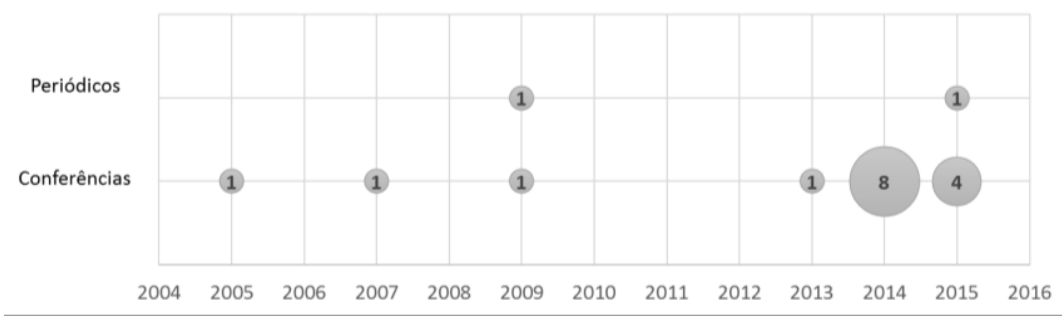

Figura 2. Distribuição das publicações por ano e por tipo de publicação. 


\subsection{Que modelos e métodos têm sido utilizados para a estimativa de tamanho e esforço de aplicativos móveis?}

Foram identificados 11 modelos de estimativa de projetos de aplicativos móveis. Podese observar que modelos de estimativa de tamanho foram os mais citados com $45 \%$, enquanto que os modelos de estimativa de esforço com $27 \%$ e os modelos que estimam tamanho e esforço em conjunto correspondem $27 \%$ também. Os resultados dessa questão de pesquisa foram subdividos em modelos de estimativa de tamanho, esforço e ambos.

- Estimativa de Tamanho - o modelo é focado em estimar o tamanho funcional do aplicativo em pontos de função [Albrecht 1979] ou pontos de função cosmic [COSMIC 2015]. Os modelos são encontrados em Abdullah et al. (2013), Abdullah et al. (2014), Heeringen e Gorb (2014), Ferrucci et al. (2015a) e D'Avanzo et al. (2015).

- Estimativa de Esforço - o modelo é focado em estimar o esforço que será necessário para desenvolver o aplicativo móvel em horas/ pontos de função cosmic. Os modelos são encontrados em Pocatilu e Ventrici (2009a), Pocatilu e Ventrici (2009b) e Keränen e Abrahamsson (2005).

- Ambos - o modelo é focado em estimar conjuntamente o tamanho e esforço para desenvolver o aplicativo móvel. Os modelos são encontrados em Nitze et al. (2014), De Souza e Aquino (2015) e Francese et al. (2015).

O resumo de cada modelo encontra-se no relatório técnico em De Souza e Conte (2017). Além disso, algumas características dos modelos julgadas relevantes foram analisadas a seguir:

- Classificação de Estimativa - os modelos encontrados foram classificados em três categorias de estimativa definidos por Mendes (2014). As categorias são modelos classificados como técnicas algorítmicas, baseado por especialista e híbridos (como por exemplo, Pocatilu e Ventrici (2009b) utiliza um modelo que é classificado em técnicas algorítmicas e baseado por especialistas). Cerca de $64 \%$ modelos são classificados como técnicas algorítmicas, 27\% híbridos e apenas $9 \%$ baseado por especialistas.

- Entrada de Dados - os modelos apontados possuem entrada de dados especificas para estimar projetos. Cerca de $18 \%$ dos modelos utilizam diagramas da UML, 64\% usam documentos de requisitos, apenas 9\% usam templete de mockups e 9\% usam uses stores. Logo a maioria utiliza documento de requisitos para estimar esse tipo de projeto.

- Métrica de Tamanho - os modelos encontrados que estimam somente o tamanho e esforço conjuntamente contabilizam 9 modelos e definiram a métrica de tamanho em pontos de função cosmic [COSMIC 2015], pontos de função [Albrecht 1979], medição RAD (documento de análise de requisitos) e medição SC (código-fonte) [FRANCESE et al 2015]. Cerca de 78\% utilizam pontos de função cosmic, $11 \%$ pontos de função, e $11 \%$ medição RAD \& medição SC. 
- Métrica de Esforço - os modelos que estimam somente o esforço conjuntamente com o tamanho contabilizam 6 modelos e utilizam as seguintes nomenclaturas para as métricas de esforço: cerca de 33\% utiliza horas, $17 \%$ pessoa-hora, $33 \%$ homens-ano e $17 \%$ não informam a métrica que utiliza.

- Ponderam Fatores - os modelos utilizam fatores preditores de esforço para estimar projetos de aplicativos móveis. Esses fatores foram classificados em Métricas de Tamanho e Fatores de Custo que são detalhados na seção 4.4. Cerca de $73 \%$ dos modelos ponderam fatores preditores de esforço específicos para aplicativos móveis que podem ser encontrados em Abdullah et al. (2013), Abdullah et al. (2014), Heeringen e Gorb (2014), Nitze et al. (2014), De Souza e Aquino (2015), Pocatilu e Ventrici (2009a), Pocatilu e Ventrici (2009b) e Francese et al. (2015). Os outros $27 \%$ dos modelos não ponderam fatores.

\subsection{Como foram avaliados os modelos e métodos de estimativa?}

De 11 modelos de estimativa de projetos de aplicativos móveis identificados, apenas 7 foram avaliados experimentalmente, correspondendo cerca de $64 \%$ dos modelos encontrados.

Apenas um estudo comparativo, realizado por Ferrucci et al. (2015b), foi encontrado no mapeamento. Eles compararam dois modelos de estimativa de tamanho proposto por D'Avanzo et al. (2015) e Heeringen e Gorb (2014). Os resultados mostraram que o tamanho funcional COSMIC [COSMIC 2015] avaliado com o modelo Heeringen e Gorb (2014) que a precisão da predição não satisfez os critérios de avaliação e se mostrou ligeiramente pior do que o obtido no estudo original baseado na abordagem proposta por D'Avanzo et al. (2015). A Tabela 3 mostra como foram avaliados experimentalmente os modelos.

Tabela 3. Lista de Modelos Avaliados Experimentalmente

\begin{tabular}{|l|l|l|l|l|}
\hline Referência & Metodologia & Ambiente & $\begin{array}{l}\text { Tipo de Análise de } \\
\text { Dados }\end{array}$ & Amostra \\
\hline Abdullah et al. $(2013)$ & Estudo de Caso & Industria & Quantitativo & 1 \\
\hline D'Avanzo et al. $(2015)$ & $\begin{array}{l}\text { Experimento } \\
\text { Controlado }\end{array}$ & Industria & Quantitativo & 8 \\
\hline Ferrucci et al. $(2015)(a)$ & Estudo de Caso & Industria & Quantitativo & 13 \\
\hline Ferrucci et al. $(2015 b)$ & $\begin{array}{l}\text { Experimento } \\
\text { Controlado }\end{array}$ & Industria & Quantitativo & 13 \\
\hline Francese et al. $(2015)$ & Estudo de Caso & Academia & Quantitativo & 23 \\
\hline Keränen e Abrahamsson (2005) & Estudo de Caso & Industria & Quantitativo e Qual. & 1 \\
\hline $\begin{array}{l}\text { De Souza e Aquino (2014 a,b,c) e } \\
\text { De Souza e Aquino (2015) }\end{array}$ & $\begin{array}{l}\text { Experimento } \\
\text { Controlado }\end{array}$ & Academia & Quantitativo & 2 \\
\hline
\end{tabular}

Os principais resultados estão destacados a seguir:

- Metodologia Experimental - os modelos foram avaliados da seguinte forma: cerca de $57 \%$ usaram estudo de caso e $43 \%$ usaram experimento controlado.

- Ambiente - cerca de $29 \%$ dos modelos foram avaliados na academia e $71 \%$ foram avaliados na indústria. 
- Tipo de Análise de Dados - os modelos foram avaliados pelos seguintes tipos de análise de dados: Cerca de $86 \%$ quantitativamente e $14 \%$ qualitativamente.

- Amostra - foi contado o número de aplicativos que foram usados para o experimento que variou entre 1 a 23 aplicativos.

\subsection{Que fatores têm sido investigados como preditores de esforço para Aplicativos Móveis?}

Entre os estudos selecionados, foram encontradas evidências relevantes de fatores preditores de esforço de projeto de aplicativos móveis. Dos 18 estudos retornados, apenas 10 apontaram fatores preditores de esforço, correspondendo a cerca de $59 \%$. Foram extraídos 93 fatores que foram organizados em uma hierarquia de 4 níveis (ver Figura 3, adaptação da hierarquia da taxonomia proposta por Britto et al. (2017), cada nível é formado por categorias.

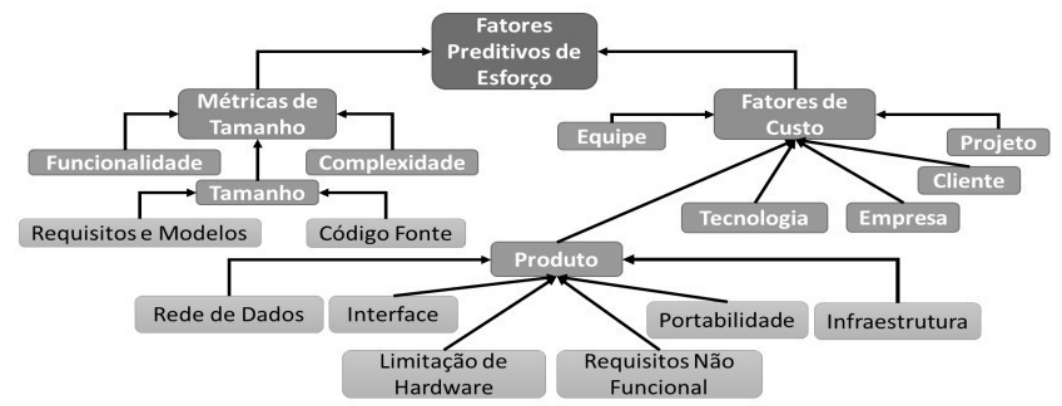

Figura 3. Fatores Preditores de Esforço: Como estão organizados

As categorias do terceiro e quarto níveis hierárquico são definidas da seguinte forma:

\section{- Métricas de tamanho}

- Tamanho - Esta categoria inclui métricas que medem diretamente o comprimento de aplicações Web com base no tamanho / comprimento dos seus elementos de composição.

- Funcionalidade - Esta categoria engloba métricas que medem indiretamente o tamanho do aplicativo móvel, baseadas em suas características e funções.

- Complexidade - Esta categoria abrange métricas que medem indiretamente o tamanho do aplicativo móvel, baseadas na dificuldade associada com os seus elementos de composição.

\section{- Fatores de custo}

- Cliente - esta categoria abrange fatores de custo relacionados ao cliente que exige o desenvolvimento de um aplicativo móvel.

- Empresa - esta categoria engloba os fatores de custos associados à empresa contratada por um cliente para desenvolver um aplicativo o aplicativo móvel.

- Produto - esta categoria inclui os fatores de custo relacionados a requisitos e restrições associada a um aplicativo móvel. 
- Projeto - esta categoria abrange os fatores de custo associados à configuração de um aplicativo móvel.

- Equipe - esta categoria abrange os fatores de custo que estão associados com a equipe de desenvolvimento responsável pela execução de um projeto de aplicativo móvel.

- Tecnologia - Esta categoria abrange os fatores de custos associados às tecnologias (Linguagem de programação, ferramentas, plataformas) exigidos em um projeto de aplicativo móvel.

Os fatores preditores de esforço estão no Apêndice deste artigo.

\section{Discussão}

Este mapeamento sistemático visou identificar modelos de estimativa e fatores preditores de esforço no contexto do desenvolvimento de aplicativos móveis. Os resultados mostraram que existem vários estudos propondo modelos e fatores preditores. No entanto, ainda existem algumas lacunas que podem ser exploradas por estudos futuros:

- Não houve consenso sobre quais fatores preditores de esforço utilizar no processo de estimativa, ou seja, apenas alguns fatores são comuns em vários estudos. Logo, uma investigação mais detalhada sobre os preditores de esforço é justificada;

- Apenas 1 estudo apresentou uma comparação entre dois modelos [FERRUCCI ET AL. 2015b], o que dificulta a definição do modelo mais adequado para o contexto de aplicativos móveis. Outro fator limitante é a indisponibilidade dos modelos em um número considerável de estudos, o que dificulta a realização desse tipo de comparação;

- Quando se trata dos modelos de estimativa mais utilizados, os resultados mostraram que há uma tendência dos pesquisadores em proporem modelos do tipo técnicas algorítmicas para estimar projetos de aplicativos móveis.

- Alguns modelos foram avaliados experimentalmente, de 11 modelos encontrados, apenas 7 foram avaliados. Dos que foram avaliados, apenas 1 foi analisado qualitativamente. Segundo Usman et al. (2014), a não medição da precisão da previsão dos modelos pode implicar no não interesse dos pesquisadores e profissionais no uso dos modelos, uma vez que não conseguiram fornecer evidência de quão adequadas são os modelos de estimativa de esforço.

- Poucos modelos estimam o tamanho e esforço conjuntamente. De 11 modelos encontrados, apenas 3 foram propostos. Modelos que estimam o tamanho e esforço em conjunto torna mais simples a estimativa.

- Atualmente, não há diretrizes para os profissionais decidirem qual modelo de estimativa ou fatores preditores de esforço escolherem em um determinado projeto de aplicativo móvel. 
Apesar da maioria dos modelos de estimativa utilizarem fatores preditores de esforço, ainda há espaço para mais pesquisas nesta área, a fim de melhorar a qualidade dos modelos de estimativa.

\section{Conclusões e Trabalhos Futuros}

Neste mapeamento sistemático, foram analisadas as publicações referentes aos modelos de estimativa de tamanho, esforço e fatores preditores de esforço de projetos de aplicativos móveis. A partir de um conjunto inicial de 487 publicações, foram selecionadas 18 publicações, resultando em 6 modelos para estimar tamanho, 3 modelos de esforço, 3 modelos para estimar ambos em conjunto e 93 fatores preditores de esforço.

Embora vários estudos tenham sido conduzidos com relação à estimativa de tamanho e esforço para projetos de aplicativos móveis, os resultados revelaram que não há um modelo contendo todos fatores citados na literatura que ajude os profissionais a selecionarem preditores de esforço. Assim, as lacunas identificadas neste mapeamento sistemático podem ser um ponto de partida para outros pesquisadores. Como trabalhos futuros pretende-se investigar qualitativamente quais são os fatores preditores de esforço que os especialistas e praticantes apontam hoje em dia na indústria e em seguida, comparar esses fatores resultantes dessas duas investigações (MSL e Qualitativo). Através desta comparação, pretende-se criar um conjunto mais completo de fatores preditores de esforço que servirá como base para uma taxonomia de fatores preditores de esforço para projetos de aplicativos móveis.

\section{Agradecimentos}

As autoras gostariam de agradecer a pesquisadora Emilia Mendes, a agência de fomento FAPEAM através do número de processo 062.02646/2014 e ao Grupo Usabilidade e Engenharia de Software (USES) da Universidade Federal do Amazonas (UFAM) por todo apoio a este trabalho.

\section{Referencias}

Abdullah, N. A. S., Rusli, N. I. A., e Ibrahim, M. F. (2013). "A case study in COSMIC functional size measurement: Angry bird mobile application”, In 2013 IEEE Conference on Open Systems, ICOS 2013, p. 139-144.

Abdullah, N. A. S., Rusli, N. I. A., e Ibrahim, M. F. (2014). "Mobile game size estimation: Cosmic fsm rules, uml mapping model and unity3d game engine". In Open Systems (ICOS), p. 42-47.

Abreu, F. P. (2011). "Estimativa de Software Baseada em Ponto de Caso de Uso: Curso introdutório", http://pt.slideshare.net/enovar/estimativa-de-software-em-pontos-decaso-de-uso, Outubro.

Albrecht, A. J. (1979). "Measuring application development productivity". In Proc. of the Joint SHARE/GUIDE/IBM Application Development Symposium, p. 83-92.

Boehm, B. W. (1981). "Software engineering economics", Vol. 197, Englewood Cliffs (NJ): Prentice-hall. 
Britto, R., Usman, M., Mendes, E. (2017). “A Taxonomy of Web Effort Predictors”, In Journal of Web Engineering (JWE).

Cohen, J., (1960). “A coefficient of agreement of nominal scales". Educational and Psychological Measurement, p.37-46.

COSMIC. (2015). "The COSMIC Functional Size Measurement Method Version 4.0.1 Measurement Manual", http://cosmic-sizing.org/publications/measurement-manual401/, Abril.

D’Avanzo, L. D., Ferrucci, F., Gravino, C., Salza, P., Giovanni, V., Ii, P., e Sa, F. (2015). "COSMIC Functional Measurement of Mobile Applications and Code Size Estimation", p. 1631-1636.

De Souza, E. T. B. e Conte, T. (2017). "Estimativa de Projetos de Aplicativos Móveis: Um Mapeamento Sistemático". In Relatório Técnico USES TR-USES-2017-011. http://uses.icomp.ufam.edu.br/wp-content/uploads/2017/05/RT-USES-2017-011.pdf.

De Souza, L. S., e De Aquino Jr, G. S. (2014). "Meffortmob: a effort size measurement for mobile application development". In: International Journal of Software Engineering and Applications, v.5, $\mathrm{n}^{\circ} 4$, p. 63.

Dombroviak, K. M., \& Ramnath, R. (2007). "A taxonomy of mobile and pervasive applications". In Proceedings of the 2007 ACM symposium on Applied computing, p. 1609-1615.

eMarketer. (2016). "2 Billion Consumers Worldwide to Get Smart(phones)", http://www.emarketer.com/Article/2-Billion-Consumers-Worldwide-Smartphonesby-2016/1011694, Abril.

Ferrucci, F., Gravino, C., Salza, P., e Sarro, F. (2015). "Investigating Functional and Code Size Measures for Mobile Applications". In 2015 41st Euromicro Conference on Software Engineering and Advanced Applications, p. 365-368.

Ferrucci, F., Gravino, C., Salza, P., e Sarro, F. (2015). "Investigating Functional and Code Size Measures for Mobile Applications: A Replicated Study". In International Conference on Product-Focused Software Process Improvement, p. 271-287.

Francese, R., Gravino, C., Risi, M., Scanniello, G., e Tortora, G. (2015). "On the Use of Requirements Measures to Predict Software Project and Product Measures in the Context of Android Mobile Apps: A Preliminary Study”. In 2015 41st Euromicro Conference on Software Engineering and Advanced Applications, p. 357-364.

Heeringen, H. Van, e Gorp, E. Van. (2014). "Measure the functional size of a mobile app: Using the cosmic functional size measurement method". Proceedings - 2014 Joint Conference of the International Workshop on Software Measurement, IWSM 2014 and the International Conference on Software Process and Product Measurement, Mensura 2014, p. 11-16.

Keränen, H., e Abrahamsson, P. (2005). "A Case Study on Naked Objects in Agile Software Development", p. 189-197. 
Kitchenham, B., e Charters, S. (2007). Guidelines for performing Systematic Literature Reviews in Software Engineering. Staffordshire, UK.

Landis, J.R. e Koch, G.G., (1977). "The Measurement of Observer Agreement for Categorical Data. Biometrics, p. 159-174.

Mendes, E. (2014). "Practitioner's Knowledge Representation: A Pathway to Improve Software Effort Estimation". Springer Science and Business, p.27.

Nagappan, M. (2016). "Future Trends in Software Engineering Research for Mobile Apps”. Software Analysis, Evolution, and Reengineering (SANER '16), p. 14-18.

Nitze, A., Schmietendorf, A., e Dumke, R. (2014). “An analogy-based effort estimation approach for mobile application development projects". Conference on Software Process and Product Measurement (IWSM-MENSURA), p. 99-103.

Pocatilu, P., e Vetrici, M. (2009). "M-applications Development using High Performance Project Management Techniques 3 Time / Duration Management Models", p. 123-128.

Trindade, C. C., Moraes, A. K., e Meira, S. R. L. (2008). "Comunicação em equipes distribuídas de desenvolvimento de software: Revisão sistemática”. In ESELAW'08: Proceedings of the 5th Experimental Software Engineering Latin American Workshop.

Usman, M., Mendes, E., Weidt, F., \& Britto, R. (2014). "Effort estimation in agile software development: a systematic literature review". In Proceedings of the 10th International Conference on Predictive Models in Software Engineering, p. 82-91.

\section{Apêndice}

A Referência EP1 a EP15 estão descritas no Apêndice B do Relatório Técnico em De Souza e Conte (2017).

\begin{tabular}{|c|c|c|c|}
\hline Métricas & Categoria & Fator & Referência \\
\hline \multirow{17}{*}{$\begin{array}{l}\text { Métricas } \\
\text { de } \\
\text { Tamanho }\end{array}$} & \multicolumn{3}{|c|}{ Tamanho } \\
\hline & \multirow{5}{*}{$\begin{array}{l}\text { Requisitos e } \\
\text { Modelos }\end{array}$} & Número de Requisitos Funcionais & EP1 \\
\hline & & Número de Atores do Caso de Uso & EP1 \\
\hline & & Número de Casos de Uso & EP1 \\
\hline & & Número de Classes & EP1 \\
\hline & & Número de Diagramas de Sequência & EP1 \\
\hline & \multirow[t]{9}{*}{ Código Fonte } & Número de Classes & EP1 \\
\hline & & Número de Arquivos & EP1 \\
\hline & & Número de Métodos (incluindo os herdados) & EP1 \\
\hline & & Número de todas as linhas & EP1 \\
\hline & & Número de linhas contendo código fonte & EP1 \\
\hline & & Número de linhas contendo comentários & EP1 \\
\hline & & Número de declarações & EP1 \\
\hline & & Tamanho do código $(\mathrm{kB})$ & EP11 \\
\hline & & $\begin{array}{l}\text { Número de arquivos XMI sobre elementos gráficos de } \\
\text { um aplicativo para dispositivos móveis. }\end{array}$ & EP1 \\
\hline & \multirow[t]{2}{*}{ Complexidade } & Complexidade & EP5 \\
\hline & & Complexidade Ciclomática & EP1 \\
\hline
\end{tabular}




\begin{tabular}{|c|c|c|c|}
\hline Métricas & Categoria & Fator & Referência \\
\hline & Funcionalidade & COSMIC & $\begin{array}{l}\text { EP3, EP4, } \\
\text { EP7, EP11, } \\
\text { EP12, EP13, } \\
\text { EP14, EP15 }\end{array}$ \\
\hline & & IFPUG & EP2 \\
\hline \multirow{39}{*}{$\begin{array}{l}\text { Fatores } \\
\text { de Custo }\end{array}$} & \multirow{8}{*}{$\begin{array}{c}\text { Equipe de } \\
\text { Desenvolviment } \\
\text { o }\end{array}$} & Produtividade da equipe & EP6 \\
\hline & & Comunicação entre a equipe & EP5, EP6 \\
\hline & & Experiência da equipe & EP6, EP7 \\
\hline & & Envolvimento individual dos membros da equipe. & EP6 \\
\hline & & $\begin{array}{l}\text { Conhecimento de desenvolvimento em áreas e aplicações } \\
\text { empresariais }\end{array}$ & EP6 \\
\hline & & Educação de pessoas & EP6 \\
\hline & & Definição de papéis das equipes & EP6, EP7 \\
\hline & & Nível de mudanças de Equipe & EP7 \\
\hline & \multirow[t]{9}{*}{ Empresa } & Organização de Domínio de Negócios & EP7 \\
\hline & & Dados de Domínio de Negócios Warehouse \& BI & EP7 \\
\hline & & Nível de certificação da empresa & EP6 \\
\hline & & Regras e Regulamentos dirigidos & EP7 \\
\hline & & Objetivos das partes interessadas. & EP6 \\
\hline & & Cliente de Domínio Empresarial e Gestão de Contas & EP7 \\
\hline & & Hipotecas de domínio empresarial & EP7 \\
\hline & & Falhas de software e hardware & EP6 \\
\hline & & Envolvimento dos concorrentes & EP6 \\
\hline & Cliente & Competências e expectativas dos usuários e clientes & EP6 \\
\hline & Fornecedor & Competências de fornecedores & EP6 \\
\hline & \multirow[t]{15}{*}{ Projeto } & Preparação da gestão da mudança & EP6 \\
\hline & & Steady Heartbeat & EP7 \\
\hline & & Processo de validação e verificação e implementação & EP6 \\
\hline & & Agile (Scrum) & EP7 \\
\hline & & Teste em ambiente real & EP6 \\
\hline & & Baseado em Release (um aplicativo) & EP7 \\
\hline & & Migração & EP7 \\
\hline & & $\begin{array}{l}\text { Nenhuma informação disponível no Campo de aplicação } \\
\text { (especialmente para novos, Aplicações inovadoras) }\end{array}$ & EP6 \\
\hline & & Tecnologia orientada (Tecnology Driven) & EP7 \\
\hline & & Versão única do Projeto & EP7 \\
\hline & & Dificuldade de mensurar o processo & EP5 \\
\hline & & Dificuldade de enumerar os estados do programa & EP5 \\
\hline & & Dificuldade de entendimento dos custos de modificações & EP5 \\
\hline & & Falta de agendar atrasos no cronograma & EP5 \\
\hline & & Falta de Confiabilidade & EP5 \\
\hline & \multirow[t]{5}{*}{ Tecnologia } & Tempo de vida que a instância da aplicação & EP8 \\
\hline & & Persistência dos dados da aplicação & EP8 \\
\hline & & Tempo de Vida & EP8 \\
\hline & & Fator de Contexto & EP2 \\
\hline & & Consciência de Localização Absoluta & EP8 \\
\hline
\end{tabular}




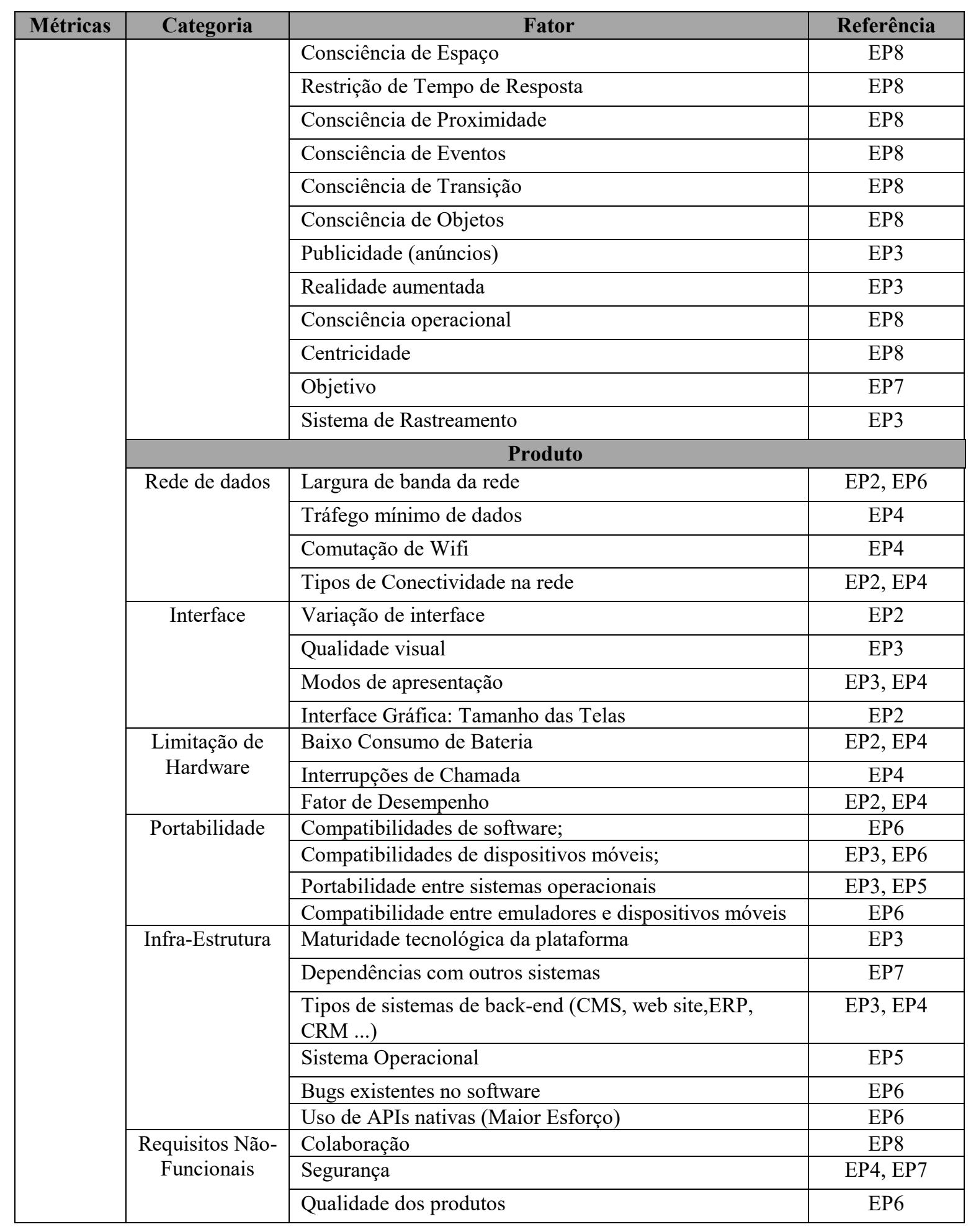

